www.jmscr.igmpublication.org

Index Copernicus Value: 79.54

ISSN (e)-2347-176x ISSN (p) 2455-0450

crossref DOI: https://dx.doi.org/10.18535/jmscr/v7i5.86

\title{
A Comparative Study of Various Screening Algorithms in the Diagnosis of Syphilis
}

\author{
Authors \\ Thilakavathi Natesan $^{1^{*}}$, V. Sudha ${ }^{2}$ \\ ${ }^{1}$ Associate Professor, Department of Serology, Madras Medical College, India \\ ${ }^{2}$ Former, Director Institute of Venereology, Madras Medical College, Chennai, India
}

\begin{abstract}
Introduction: Worldwide two strategies are followed to diagnose syphilis. One is conventional method of testing the serum by non-specific treponemal test VDRL followed by specific Treponemal Pallidum Hemagglutination Assay (TPHA). The other method is viceversa. We aim to study the percentage of syphilis case detection rate by both the methods.

Material and Methods: A retrospective cross-sectional study was conducted amongst the high risk group patients attending the STD-OP of Institute of Venereology, Chennai for a period of 12 months. The study population was divided into 2 groups. Group A with conventional screening method (i.e. VDRL followed by TPHA) and Group B with TPHA followed by VDRL.

Results: A total of 1527 cases were recruited into the study, 767 in Group A and 760 in Group B. Group A: Out of 767 cases, 90 (11.7\%) were reactive by VDRL. 65 (72.2\%) were TPHA positive among the 90 VDRL reactive. The case detection rate and correlation between the two tests were $11.7 \%$ and $72.2 \%$ respectively. Group B: Out of 760 cases, 263 (34.6\%) were TPHA positive 166 were VDRL reactive among the TPHA positive. The case detection rate and correlation between the two tests were $34.6 \%$ and $63.1 \%$ respectively.

Conclusion: The results of comparative data clearly demonstrate that the case detection rate was higher (three times) in reverse screening than that of conventional screening method. In conclusion, consistent with recommendations, laboratories should consider implementing the reverse algorithm for the diagnosis of syphiilis.
\end{abstract}

\section{Introduction \& Background}

In developing countries, STIs (Sexually Transmitted Infections) and their complications are amongst the top five disease categories for which adults seek health care. Everyday nearly 1 million people acquire a new STI, and more than 340million new cases of curable STIs occur throughout the world each year. ${ }^{1}$ Syphilis constitute a major percentage of curable STI and this infection can also be passed on from a mother to her fetus during pregnancy. As a cause of genital ulcer disease, syphilis has been associated with an increased risk of HIV transmission and acquisition. Clinical diagnosis of Syphilis is complex and often missed because of its latency, typical\& asymptomatic clinical presentation. Serological diagnosis using VDRL (Venereal Disease Research Laboratory) a non-specific test and TPHA (Treponema pallidum hemagglutination) a specific test plays a vital role in the diagnosis of syphilis. In recent years some clinical laboratories have implemented are verse 
screening algorithm in the diagnosis of syphilis ${ }^{2}$ (specific treponemal tests followed by nontreponemal tests). But most of the laboratories follow the traditional approach of testing (nonspecific treponemal tests followed specific treponemal tests). These two algorithms have created some confusion among health care providers. Hence this study was undertaken to find out the case detection rate and concordance between the two tests in a given sample by both methods.

\section{Materials \& Methods}

The present study involved the case detection rate using TPHA and VDRL by conventional and the reverse screening method. The study period was between January 2012 and December 2012. The samples were collected from the individuals attending the STD-OP of tertiary care centre with the history of premarital, extramarital exposure, multiple partners, intravenous drug abuser, homosexuals, trans genders and female sex workers (FSW) were included in the study. The study population was divided into two groups Group A and Group B. The study samples were recruited into each group sequentially. In Group A conventional screening algorithm (VDRL followed by TPHA) and in Group B reverse screening algorithm was adopted.

\section{Results}

The test results of conventional \& reverse screening algorithm were shown in Figs. 1 and 2. 1527 samples were recruited into the study, 767 in Group A and 760 in Group B (see Fig. 2).

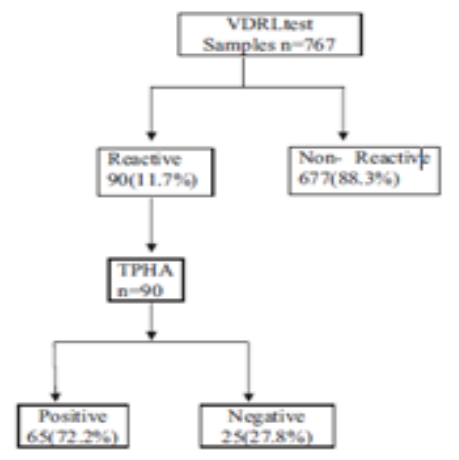

Fig. 1 e Group A conventional screening algorithm.

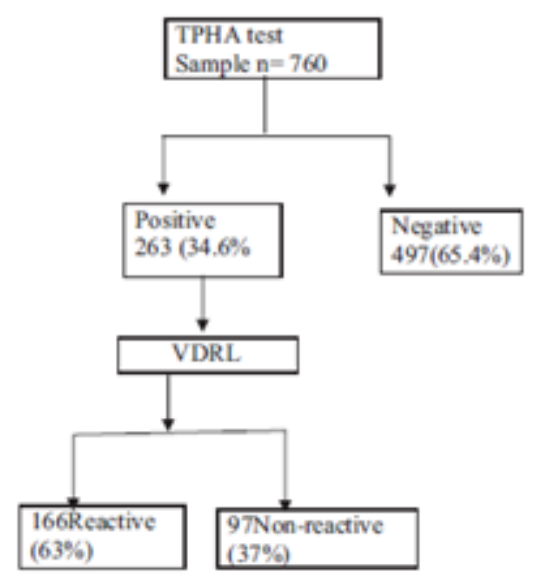

Fig. 2 e Group B reverse screening algorithm 3.1. Group A

Out of 767 samples e 90 samples were reactive by VDRL. The case detection rate is $11.7 \%$. The reactive samples by VDRL were subjected to TPHA. Out of 90 cases, 65 (72.2\%) samples showed positive results and $25(27.8 \%)$ samples showed negative results for TPHA test. The percentage of concordance between the VDRL and TPHA by conventional method was $72.2 \%$.

\section{Group B}

Out of 760 samples 263 were positive by the specific TPHA test. The case detection rate by reverse screening method was $34.6 \%$. Out of 263 TPHA positive samples $166(63 \%)$ were reactive and $97(37 \%)$ were non reactive by VDRL. The percentage of concordance between the VDRL and TPHA by reverse screening method was $63 \%$.

\section{Discussion}

The case detection rate in the conventional method is $11.8 \%$, when compared to reverse method e $34.6 \%$. Our study shows that the case detection rate was higher by 3 times in the reverse screening method. Our study correlates with the study of Huh-HJ et al (2007). Study by Huh HJ, Lee KK, Kim ES, ChaeS3 showed RPR a nonspecific tests the positive rate is $0.23 \%$ and in the specific test the case detection rate is $1.6 \%$ in the general population. The case detection rate in their study was increased by 5 times while following the reverse screening method. Also Binicker et al, 2012 has clearly stated that screening for syphilis 
using treponemal assay detects a higher number of patients with reactive results compared to traditional screening by non-treponemal tests. ${ }^{4}$ The higher case detection rate by reverse method (TPHA) may be attributed due to the use of specific antigen, automation of tests, capacity for detecting in early, latent cases, past, treated and untreated syphilis. The lower case detection rate of non-specific test may be attributed to the fact that it is reactive when the disease activity is high (i.e.) secondary and tertiary syphilis missing few cases of primary, latent syphilis, false negative due toprozone phenomenon and technical errors. The use of treponemal immunoassays to screen for syphilis eliminates biological false positives due to the presence of anticardiolipin antibodies from other diseases. A number of studies have shown that specific treponemal immunoassays have fewer false positives than non treponemal assays. ${ }^{5 \mathrm{e} 7}$ Rare false negative immunoassay results during early primary syphilis can be mitigated. After the introduction of reverse sequence syphilis screening in Alberta, Canada, there was an increase in the diagnosis of late latent syphilis in individuals screening positive with the treponemal test. The concordance in the conventional method was $(72 \%)$ between VDRL and TPHA. This discordance may be due to biological false positive reactions or due to technical errors. In the reverse screening method out of 760 samples 166 were positive by both TPHA and VDRL. 97 (37\%) samples were TPHA positive, VDRL negative (75 cases had a history of treated syphilis). The concordance between TPHA and VDRL63\% this kind of situation may arise in fully treated patients orlatent or late syphilis, false negative due to VDRL (Prozone phenomenon \& technical error). Non treponimal test has less sensitivity in late stage of syphilis. The titre of Cardiolipin antibodies may be less in latent syphilis. In the UK considering the sensitivity and specificity of treponemal test have proposed that either TPHA alone or a combination of VDRL/RPR tests and TPHA/TPPA can be used for syphilis screening.

\section{Conclusion}

The results of comparative data clearly demonstrate that the case detection rate was higher (three times) in reverse screening than the conventional screening method. In conclusion, consistent with recommendations, ${ }^{8 \mathrm{e} 10}$ laboratories should consider implementing the reverse algorithm for the diagnosis of syphilis.

\section{Conflicts of Interest}

All authors have none to declare.

\section{Acknowledgment}

I acknowledge the help received from Dr. Khusboo mini, Mrs. S. Radha, Mrs. S. Geetha, Mrs. V. Shanmugapriya \& Ms. M.Kalaivani, Department of Serology, Institute of Venereology, Madras Medical College, Chennai-03.

\section{References}

1. Global prevalence and Incidence of selected curable sexually transmitted infections Overview and estimates and estimates WHO/HIV_AIDS/2001.02 WHO/CDS/CSR/EDC/2001.10.

2. Lipinsky Dafna, Schreiber Licita, Kopel Vered, Shainberg Bracha. Validation of reverse sequence screening for syphilis.J Clin Microbiol. 2012 April;50:1501. http://dx.doi.org/10.1128/JCM.06286-11.

3. Huh HJ, Lee KK, Kim ES, Chae SL. Analysis of positive resultsin mediace rapid plasma reagin and Treponema pallidum latex agglutination as the automated syphilis test. Korean J LabMed. 2007 Oct;27:324e329.

4. Binnicker MJ, Jespersen DJ, Rollins LO. Direct comparison ofthe traditional and reverse syphilis screening algorithms in a population with a low prevalence of syphilis. J Clin Microbiol. 2012;50:148e 150 .

5. Loeffelholz MJ. It is time to use treponema-specific antibody screening 
tests for diagnosis of syphilis. J Clin

Microbiol. January 2012;50:2e6.

6. Marangoni A, Moroni A, Accardo S, Cevenini R. Laboratory diagnosis of syphilis with automated immunoassays. J ClinLab Anal. 2009;23:1e6.

7. Marangoni A, Sambri V, Accardo S, et al. Evaluation of LIAISON Treponema Screen, a novel recombinant antigen based chemiluminescence immunoassay for laboratory diagnosis of syphilis. Clin Diagn Lab Immunol.2005;12:1231e1234.

8. Health Protection Agency. Serological Diagnosis of Syphilis, National Standard Method, London, United Kingdom.vol. 44. 2007.Available from: http://www.hpatandardmethods.org.uk.

9. Gratrix J, Plitt S, Lee BE, et al. Impact of reverse sequence syphilis screening on new diagnoses of late latent syphilis in Edmonton, Canada. Sex Transm Dis. 2012;39:528e530.

10. Geusau A, Kittler H, Hein U, DanglErlach E, Stingl G, Tschachler E. Biological false-positive tests comprise a high proportion of Venereal Disease Research Laboratory reactionsin an analysis of 300,000 sera. Int J STD AIDS. 2005Nov;16:722e726. 